\title{
Temporal succession of soil antibiotic resistance genes following application of swine, cattle and poultry manures spiked with or without antibiotics
}

\author{
Yu-Jing Zhang a , Hang-Wei Hu ${ }^{\text {a, }{ }^{* *}, \text { Min Gou }}{ }^{\text {, }}$, Jun-Tao Wang ${ }^{\mathrm{b}}$, Deli Chen ${ }^{\mathrm{a}}$, \\ Ji-Zheng $\mathrm{He}^{\mathrm{a}, \mathrm{b}, *}$ \\ ${ }^{a}$ Faculty of Veterinary and Agricultural Science, The University of Melbourne, Parkville, VIC 3010, Australia \\ b State Key Laboratory of Urban and Regional Ecology, Research Centre for Eco-Environmental Sciences, Chinese Academy of Sciences, Beijing 100085, China
}

\section{A R T I C L E I N F O}

\section{Article history:}

Received 9 June 2017

Received in revised form

20 September 2017

Accepted 20 September 2017

Available online 28 September 2017

\section{Keywords:}

Animal manure

Tylosin

Antibiotic resistance genes

Mobile genetic elements

Public health

\begin{abstract}
A B S T R A C T
Land application of animal manure is a common agricultural practice potentially leading to dispersal and propagation of antibiotic resistance genes (ARGs) in environmental settings. However, the fate of resistome in agro-ecosystems over time following application of different manure sources has never been compared systematically. Here, soil microcosm incubation was conducted to compare effects of poultry, cattle and swine manures spiked with or without the antibiotic tylosin on the temporal changes of soil ARGs. The high-throughput quantitative PCR detected a total of 185 unique ARGs, with MacrolideLincosamide-Streptogramin B resistance as the most frequently encountered ARG type. The diversity and abundance of ARGs significantly increased following application of manure and manure spiked with tylosin, with more pronounced effects observed in the swine and poultry manure treatments than in the cattle manure treatment. The level of antibiotic resistance gradually decreased over time in all manured soils but was still significantly higher in the soils treated with swine and poultry manures than in the untreated soils after 130 days' incubation. Tylosin-amended soils consistently showed higher abundances of ARGs than soils treated with manure only, suggesting a strong selection pressure of antibioticspiked manure on soil ARGs. The relative abundance of ARGs had significantly positive correlations with integrase and transposase genes, indicative of horizontal transfer potential of ARGs in manure and tylosin treated soils. Our findings provide evidence that application of swine and poultry manures might enrich more soil ARGs than cattle manure, which necessitates the appropriate treatment of raw animal manures prior to land application to minimise the spread of environmental ARGs.
\end{abstract}

(C) 2017 Elsevier Ltd. All rights reserved.

\section{Introduction}

The increasing prevalence of antibiotic resistance genes (ARGs) in environmental settings and their potential acquisition by human pathogens has become a global public health concern in the 21st century (Rossolini et al., 2014; WHO, 2014). Antibiotic-resistant infections are now responsible for more than half a million human deaths globally each year (Dominey-Howes and Labbate,

\footnotetext{
t This paper has been recommended for acceptance by Klaus Kummerer.

* Corresponding author. Faculty of Veterinary and Agricultural Science, The University of Melbourne, Parkville, VIC 3010, Australia.

** Corresponding author.

E-mail addresses: hang-wei.hu@unimelb.edu.au (H.-W. Hu), jizheng.he@ unimelb.edu.au (J.-Z. He).
}

2014), while over 10 million deaths in 2050 is predicted if the antibiotic resistance issue is not tackled from now onwards. The common use of antibiotics in clinical environments and livestock production is considered the major cause leading to the global spread of antibiotic resistant bacteria (Sarmah et al., 2006; WHO, 2014). Environmental ARGs are subjected to potential transmission to human pathogens and commensals via the route of horizontal gene transfer (HGT; conjugation, transduction and transformation) mediated by mobile genetic elements (e.g., plasmids, integrons, and transposons) (Thomas and Nielsen, 2005; Heuer et al., 2011), or via the growth and spread of antibiotic resistant bacteria (Manaia, 2017). Therefore, ARGs have been recognized as a novel type of environmental contaminant (Pruden et al., 2006) attracting emerging efforts to understand the behaviors and mobility of ARGs in diverse environments and their links 
with clinically-relevant pathogens (Forsberg et al., 2012; Han et al., 2016; Hu et al., 2017).

The global consumption of antibiotics substantially increased from 50 billion to 70 billion standard units between 2000 and 2010, among which $70-80 \%$ are applied to livestock industry, especially in poultry, swine, and cattle husbandry (Gelband et al., 2015). Tetracyclines, macrolides, and sulfonamides are three most commonly used antibiotics in livestock, and are also widely used in human medicines for treatment of infections (Jechalke et al., 2014b; Gelband et al., 2015). Along with the increasing consumption of antibiotics, it was found that veterinary antibiotics are poorly digested in the guts of animals, and a significant amount (30-90\%) of them are released into the environment through animal urination and defecation (Zhang and Zhang, 2011). The use of veterinary antibiotics posed a strong pressure on the gut microbiomes by selecting antibiotic resistant bacteria through mutation or acquisition of ARGs (Tenover, 2006). An emerging body of studies have reported that there is a positive correlation between dosage of veterinary antibiotics and the occurrence of ARGs in manure and agricultural soils (Bibbal et al., 2007; Byrne-Bailey et al., 2009; Heuer and Smalla, 2007; Hölzel et al., 2010; Koch et al., 2017; Muurinen et al., 2017). Therefore, it is imperative to understand the impact of antibiotic usage on ARGs in veterinary settings and their fate after release into the environment.

The use of antibiotics in livestock production and the prevalence of ARGs in animal gastrointestinal tract have triggered global concerns that land application of animal manures may lead to the dissemination of ARGs in agro-ecosystems (Heuer et al., 2011; Thanner et al., 2013), even if the animals have never been treated with antibiotics (Udikoviv-Kolic et al., 2014; Hu et al., 2016a). Apart from being recognized as a rich reservoir of ARGs phylogenetically close to potential human pathogens (Heuer et al., 2011; Forsberg et al., 2012; Zhu et al., 2013), manure can also provide nutrients for favoring the occurrence of HGT and growth of ARGs-carrying bacteria (Smalla et al., 2000; Hu et al., 2016a). These manurederived ARGs are in high risk of spreading into the food chain (Marti et al., 2013; Zhu et al., 2017), and may pose a potential threat to public health when vegetables grown in manured soils are consumed by humans. Recent studies have started to shed light on the transmission of manure-derived ARGs in agricultural soils following land application of animal manures (Jechalke et al., 2013, 2014a; Garder et al., 2014; Peng et al., 2015; Hu et al., 2016a; Luby et al., 2016; Gou et al., 2017). Most of these studies, however, focused on only single type of manure or a limited number of welldocumented ARG types (Heuer and Smalla, 2007). To our knowledge, no studies have attempted to systematically compare the impacts of different sources of animal manures, in the presence or absence of antibiotics, on the temporal patterns of a broad spectrum of ARGs. An improved understanding of this knowledge is critical to prediction of ARG behaviours in soil environments, and development of appropriate manure treatment approaches to minimise the spread of environmental ARGs.

Therefore, the objective of this study is to compare the effects of amendment of three animal manure sources (swine, cattle, and poultry) on the temporal succession of a diverse array of ARGs in soil microcosms incubated with agricultural soils. Tylosin, a macrolide antibiotic used in livestock industries for growth promotion and therapy (Sarmah et al., 2006; Apley et al., 2012), was spiked into manure to test their synergistic selection effects on antibiotic resistance. The high-throughput quantitative PCR array was performed to target 285 ARGs which confer resistance to all major classes of antibiotics; and 10 mobile genetic elements (MGEs) as a proxy for HGT potential of ARGs. We hypothesized that: (i) amendment of different animal manure sources would differ in their impacts on the temporal succession of soil ARGs owing to their different intrinsic ARG profiles and selection pressure; (ii) Tylosin-spiked manure would further enhance the selection pressure on soil ARGs and their HGT potential.

\section{Materials and methods}

\subsection{Soil and manure sampling}

The soil used in this study was taken from a vegetable farm at Clyde $\left(38^{\circ} 07^{\prime} \mathrm{S}, 145^{\circ} 19^{\prime} \mathrm{E}\right)$, Victoria, Australia. The soil in this site has a pH value of 7.2 and is classified as loamy sand. Total carbon and total nitrogen are $3.75 \%$ and $0.43 \%$, respectively. Cattle manure and swine manure were collected from the Dookie Farm $\left(36^{\circ} 25^{\prime} \mathrm{S}\right.$, $145^{\circ} 42^{\prime} \mathrm{E}$ ), University of Melbourne, and poultry manure in the litter form was taken from a chicken farm in Mornington, Melbourne. Manure samples were collected within three days after excretion from animals without known history of antibiotic treatment. Soil and manure samples were kept on ice during transportation and stored at $4{ }^{\circ} \mathrm{C}$ before analysis. The antibiotic tylosin used in the experiment was purchased from Sigma-Aldrich company (St Louis, MO, USA)

\subsection{Soil microcosm incubations}

Three sets of soil microcosm incubation experiments were established depending on the amendment of different animal manure sources, and each set included two treatments (Fig. S1). In the first treatment, manure was mixed thoroughly with sieved soils $(<2 \mathrm{~mm})$ to reach a final concentration of $80 \mathrm{mg} \mathrm{g}^{-1}$ dry soil, which is corresponded to a typical agricultural amount of $60 \mathrm{~m}^{3}$ manure per hectare. In the second treatment, tylosin in aqueous solutions was spiked to the manure and the tylosin-spiked manure was mixed with soil to reach a concentration of $0.1 \mathrm{mg}$ tylosin $\mathrm{g}^{-1}$ soil and $60 \mathrm{~m}^{3}$ manure per hectare. The untreated soil sample with same water content (60\% of the water filled pore space) was used as the control treatment. Soil microcosms were established in $250 \mathrm{~mL}$ vials with $20 \mathrm{~g}$ of soil or manured soil (oven dry-weight equivalent), loosely covered, and incubated in the dark at $25^{\circ} \mathrm{C}$. The aerobic condition in the microcosms was maintained by opening the vials for air refreshing every three days. Soil moisture contents were maintained by adding sterilized water regularly. A total of 168 soil microcosms were destructively sampled at eight time points on days $1,7,20,35,50,70,100$, and 130 after manure application.

\subsection{Soil physicochemical analysis and genomic DNA extraction}

Soil total nitrogen and total carbon were measured using the classic Dumas method of combustion on the isotope-ratio mass spectrometry (Sercon Hydra, Crewe, United Kingdom). Soil pH were measured with a soil to water ratio of 1: 5 using the Orion Star A211 pH Meter (Thermo Scientific Inc., Melbourne, Australia). Soil water content was measured by oven-drying soils samples at $105{ }^{\circ} \mathrm{C}$ for $24 \mathrm{~h}$. DNA was extracted from soil and manure samples using the MoBio PowerSoil DNA extraction kit (MoBio Laboratories, Carlsbad, CA, USA) following the manufacturer's instructions. The concentration and purity of the extracted DNA were assessed using the NanoDrop ND2000c spectrophotometer (NanoDrop Technologies, Wilmington, DE, USA).

\subsection{High-throughput quantitative $P C R$}

High-throughput qPCR (HT-qPCR) was performed to determine the diversity (i.e. number of positive targets) and abundance of ARGs using the Wafergen SmartChip Real-time PCR system 
(Fremont, CA, USA). The Wafergen system is a high-throughput qPCR platform with the capability to run 5184 reactions with each volume of $100 \mathrm{~nL}$ using the SensiMix SYBR No-ROX reagent (Bioline, London, UK). Primers, DNA and reagents were dispensed into the 5184-nanowell SmartChip using a Multisample NanoDispenser (Fremont). The HT-qPCR array contained a total of 296 primer sets (Hu et al., 2016b), including 285 primer sets targeting eight major classes of ARGs, 10 primer sets targeting MGEs, and one 16S rRNA gene as the internal control (Table S1). The specificity of all primers was verified through amplicon sequencing, BLAST search and amplification efficiency check (Looft et al., 2012; Johnson et al., 2016). The SmartChip was loaded into the Wafergen SmartChip Real-Time PCR Cycler using the thermal-cycling conditions as follows: $95^{\circ} \mathrm{C}$ for $10 \mathrm{~min}$, followed by 40 cycles of $95^{\circ} \mathrm{C}$ for $30 \mathrm{~s}$ and $60{ }^{\circ} \mathrm{C}$ for $30 \mathrm{~s}$. Only well data with the amplification efficiencies within 1.7-2.3 were remained. A threshold cycle $\left(C_{T}\right)$ value of 31 was used as the detection limit (Su et al., 2014). Three technical replicates were included for each sample, and samples with all the three technical replicates above the detection limit were regarded as positive quantification. A comparative $C_{T}$ method, also referred to as the $\Delta \Delta C_{T}$ method of relative profiling, was performed to assess the temporal changes of the relative abundance of ARGs across all the samples (Schmittgen and Livak, 2008). The relative abundance of a specific ARG in a sample was calculated from the average of the relative abundances of the three technical replicates.

\subsection{Quantitative PCR ( $q P C R$ ) analysis of the bacterial $16 S$ rRNA gene}

The absolute abundance of 16S rRNA gene was determined on a Bio-Rad CFX384 Real-Time PCR Detection System (Bio-Rad, Herculers, USA) using the primer set BACT1369F/PROK1492R (Suzuki et al., 2000). Each $10 \mu \mathrm{L}$ reaction system included $5 \mu \mathrm{L}$ SensiMix SYBR No-ROX reagent (Bioline), $0.4 \mu \mathrm{L}$ of each primer $(10 \mu \mathrm{M}), 2 \mu \mathrm{L}$ DNA template, and $2.2 \mu \mathrm{L}$ nuclease-free PCR-grade water. Thermalcycling conditions were as follows: an initial enzyme activation at $95^{\circ} \mathrm{C}$ for $10 \mathrm{~min}$, followed by 40 cycles of denaturation at $95{ }^{\circ} \mathrm{C}$ for $15 \mathrm{~s}$ and a final annealing and extension at $60{ }^{\circ} \mathrm{C}$ for $1 \mathrm{~min}$. To generate standard curves for qPCR analysis, PCR amplicons of the bacterial 16S rRNA gene by using the primers BACT1369F/ PROK1492R were purified and ligated into the pGEM-T Easy vector (Promega, Madison, WI, USA), and the resultant ligation products were transformed into JM109 competent cells following the manufacturer's instructions. The resulting clones containing the targeted bacterial 16S rRNA gene fragment were selected to extract plasmid DNA, and standard curves were generated by preparing the plasmid in 10 -fold serial dilutions.

\subsection{Co-occurrence analysis and network generation}

The CoNet Cytoscape plug-in method was used to visualize the co-occurrence patterns of ARGs and MGEs in the network interface (Soffer et al., 2015). ARGs with a minimum occurrence of 3 across all soil samples from the whole microcosm experiment $(n=168)$ were regarded as poorly represented ARG subtypes and discarded to reduce artificial correlation bias. The $P$-values were calculated based on two dissimilarity methods (Bray-Curtis and KullbackLeibler) and two correlation methods (Spearman and Pearson) to control the potential of obtaining false-positive results. The significant pairwise correlations $(P$-value $<0.05)$ between the ARG and MGE subtypes were utilized to form their co-occurrence networks. The resultant co-occurrence network was visualized using the Frucherman Reingold algorithm on the interactive platform of Gephi (Bastian et al., 2009). A correlation with a correlation coefficient $(\rho$-value $)>0.8$ and a significant level $(P$-value $)<0.05$ were considered statistically robust (Junker and Schreiber, 2008).

\subsection{Statistical analysis}

One-way analysis of variance (ANOVA) with repeated measures was performed to analyse the effects of manure application and tylosin addition on the diversity, relative and absolute abundances of ARGs and MGEs, followed by Student-Newman-Keuls test to check for quantitative variance between different treatments. Pearson's correlation test was performed to test the correlations between the relative abundance of ARGs and MGEs. Non-metric multidimensional scaling (NMDS) analysis based on the relative abundance of ARGs was performed to visualize the Bray-Curtis dissimilarity matrices. The heatmap showing the overall HT-qPCR array data of ARGs with log-transformed relative abundances was generated with the "ggplot2" package in R platform. The Venn diagrams showing the unique and shared ARG subtypes across different treatments and manure sources were performed with the "gplots" package in R platform.

\section{Results}

\subsection{Diversity of ARGs and MGEs in different treatments}

The HT-qPCR array targeting 285 ARGs and 10 MGEs was performed to compare the effects of three types of animal manure with or without tylosin addition on the diversity of ARGs and MGEs (i.e. the number of detected ARGs and MGEs) in an agricultural soil. The ARGs detected in these samples encompass three major resistance mechanisms: antibiotic deactivation, efflux pump, and cellular protection, with antibiotic deactivation (39\% of total ARGs) as the most common resistance mechanism (Fig. S2). The detected ARGs can potentially confer resistance to eight major classes of antibiotics, of which MLSB (20\%) and aminoglycoside (19\%) were the two most frequently detected ARG types (Fig. 1a). Other frequently detected ARGs included multidrug (18\%), tetracycline (17\%) and $\beta$ lactamase (13\%) (Fig. 1a).

Untreated soil samples harbored the lowest number of detected ARGs $(68.8 \pm 2.7$ on average out of the 285 target ARGs, $n=24)$ among all the treatments (Fig. 1b). The average numbers of ARGs detected in poultry manure $(109.7 \pm 5.9, \mathrm{n}=3)$ and swine manure $(109.0 \pm 4.2, \mathrm{n}=3)$ were significant higher than that in cattle manure $(82.0 \pm 5.0, \mathrm{n}=3)(P<0.05)$. Among all the manure-treated soil samples without tylosin, the number of detected ARGs ranged from 58 to 133 in individual samples and a significantly higher ARG diversity was observed in poultry and swine manure-treated samples (89.9 \pm 3.1 and $101.4 \pm 2.5$, respectively, $\mathrm{n}=24$ ) compared with cattle manure-treated samples $(76.9 \pm 0.53, \mathrm{n}=24)$ (Fig. 1b, $P<0.05$ ). Tylosin-spiked cattle manure also harbored the lowest diversity of ARGs among all manure-treated samples spiked with tylosin (Fig. 1b). The average numbers of detected MGEs (CK: $4.1 \pm 0.2$; PM: $5.6 \pm 0.2$; PMA: $6.2 \pm 2.8$; CM: $5.9 \pm 0.5$; CMA: $5.9 \pm 2.4$; SM: $6.6 \pm 2.5$; SMA: $7.2 \pm 3.1$ ) were similar among all the samples but slightly higher in the manure samples (UPM: $8.0 \pm 4.2$; UCM: $8.7 \pm 2.3$; USM: $8.7 \pm 1.5$ ) (Fig. $1 \mathrm{~b}$ ).

Venn diagrams showed the number of unique and shared ARGs among different manure and soil samples (Fig. 1c and d). A total of 86 ARGs were shared among the three types of animal manures, and 22, 19 and 3 unique ARGs were observed in swine, poultry, and cattle manures, respectively (Fig. 1c). Untreated soil samples shared 129 ARGs with manure-treated samples. In this study, we observed the introduction of 32,28 , and 28 unique ARGs in soils from amendment of poultry, cattle, and swine manures, respectively (Fig. 1d). 

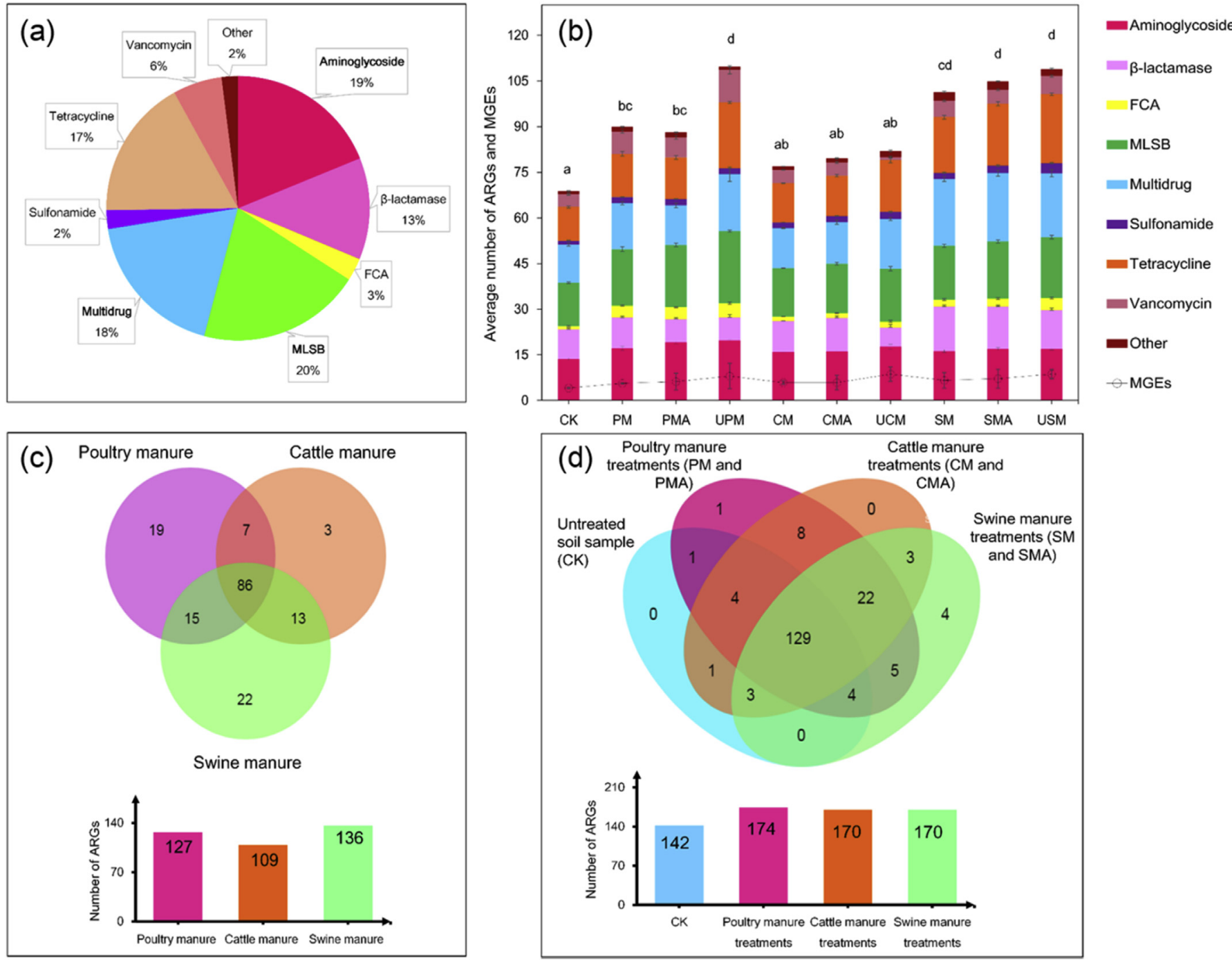

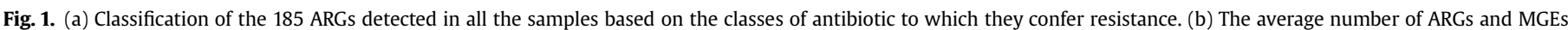

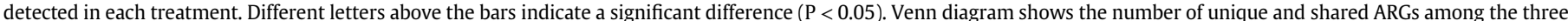

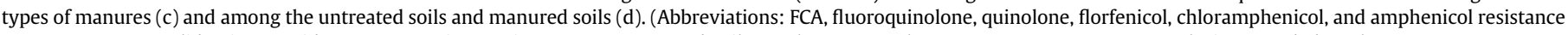

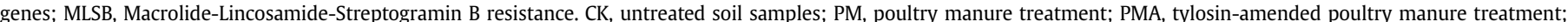

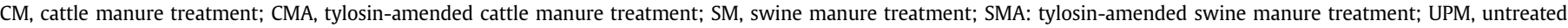
poultry manure; UCM, untreated cattle manure; USM, untreated swine manure).

\subsection{Abundance of ARGs and MGEs in different treatments}

The comparative $C_{T}$ method was used to calculate the relative abundances of ARGs and MGEs by normalizing to 16S rRNA gene in the same HT-qPCR array (Schmittgen and Livak, 2008). The relative and absolute abundances of ARGs in cattle manure samples were significantly lower compared with those in swine and poultry manure samples, with swine manure harbouring the highest abundance of ARGs (Fig. 2a and c). When animal manure was applied to the soil, the relative and absolute abundances of ARGs in soil samples treated with cattle and swine manure were significantly lower than those in poultry manure-treated samples (Fig. 2b and d). In manure-treated samples spiked with tylosin, the ARG abundances were significantly higher than those in samples without tylosin addition $(P<0.05$, Fig. $2 \mathrm{~b}$ and $\mathrm{d})$. The pattern of MGE abundances was quite similar to that of ARG abundances, with the lowest abundances found in cattle manure and cattle manuretreated soil samples (Fig. 2).

\subsection{Temporal changes of ARGs and MGEs in manure-treated soils}

A total of 168 soil microcosms were destructively sampled at eight time points (days 1, 7, 20,35, 50, 70, 100, and 130) during the incubation to explore the temporal changes of ARGs and MGEs in soils treated with three sources of animal manures with or without tylosin (Fig. 3). The ANOVA analysis with repeated measures found that the sampling time and the interaction of sampling time and treatment had significant effects on the average number, relative and absolute abundances of both ARGs and MGEs during the microcosm incubation (Table S2).

The numbers of detected ARGs in manure-treated soils significantly decresed over time during the incubation, regardless of whether or not tylosin was added $(P<0.05)$, while those in untreated soils stayed largely unchanged (Fig. 3a). Interestingly, the ARG numbers in soils treated with cattle manure approached the background levels of ARGs in untreated soils at the end of incubation (day 130), but swine and poultry manure-amended soils still 

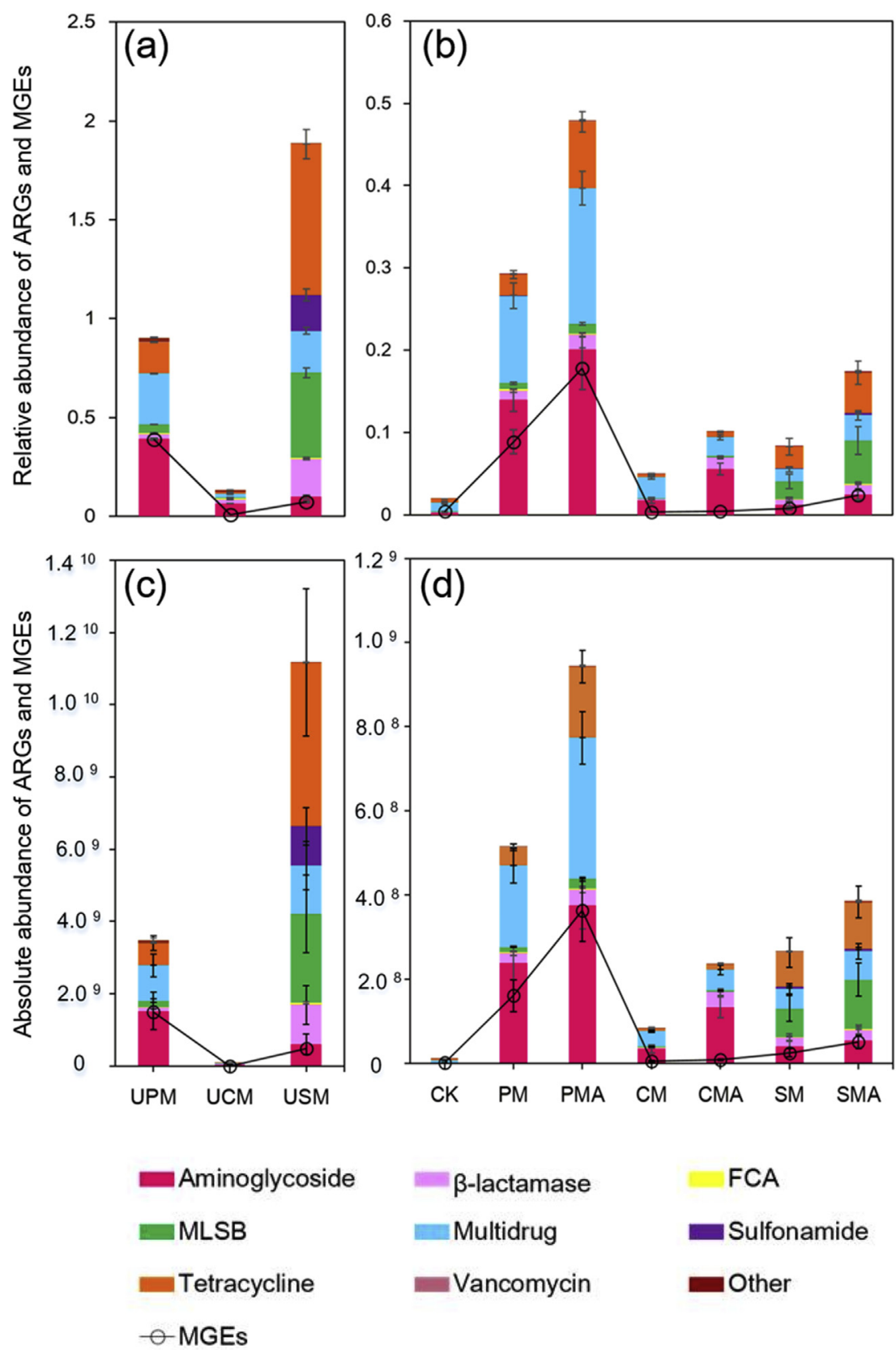

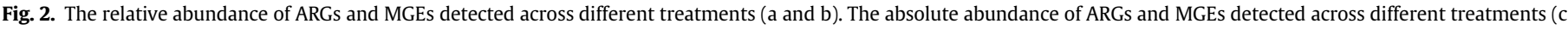

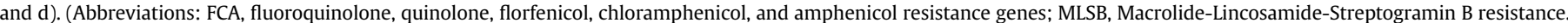

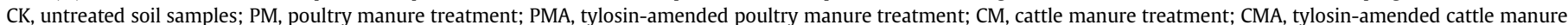

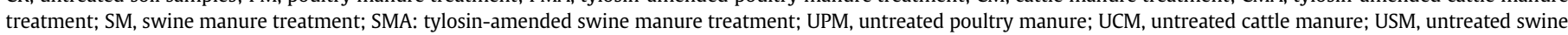
manure).

had a significantly higher level of ARGs than untreated soils at day $130(P<0.05$, Fig. 3a). The swine manure-treated soils tended to have a higher diversity of ARGs than poultry manure-treated soils, followed by cattle manure-treated soils during the incubation (Fig. 3a).

The relative abundances of ARGs in soils treated with poultry manures significantly declined over time but were still significantly higher than that in untreated soils $(P<0.05)$, with a more pronounced selection effect observed in tylosin-amended samples (Fig. 3b). The ARG abundances in soil samples treated with swine manure only or swine manure spiked with tylosin declined sharply to background levels in untreated soils within 7 or 20 days of incubation, respectively, and then remained largely unchanged till day 130 (Fig. 3b). The application of cattle manure did not significantly increase the relative abundance of soil ARGs, while the tylosin-spiked cattle manure increased ARG abundances at the initial stages of incubation and then declined to be close to the background level at day 130 . The tylosin-amended manure application showed significantly higher relative abundances of ARGs compared with their corresponding no-tylosin treatments for all the three manure sources $(P<0.05$, Fig. $3 b)$. The absolute abundances of ARGs showed similar temporal patterns as the relative abudances of ARGs during the incubation (Fig. S3).

The number of detected MGEs did not show clear trends during 

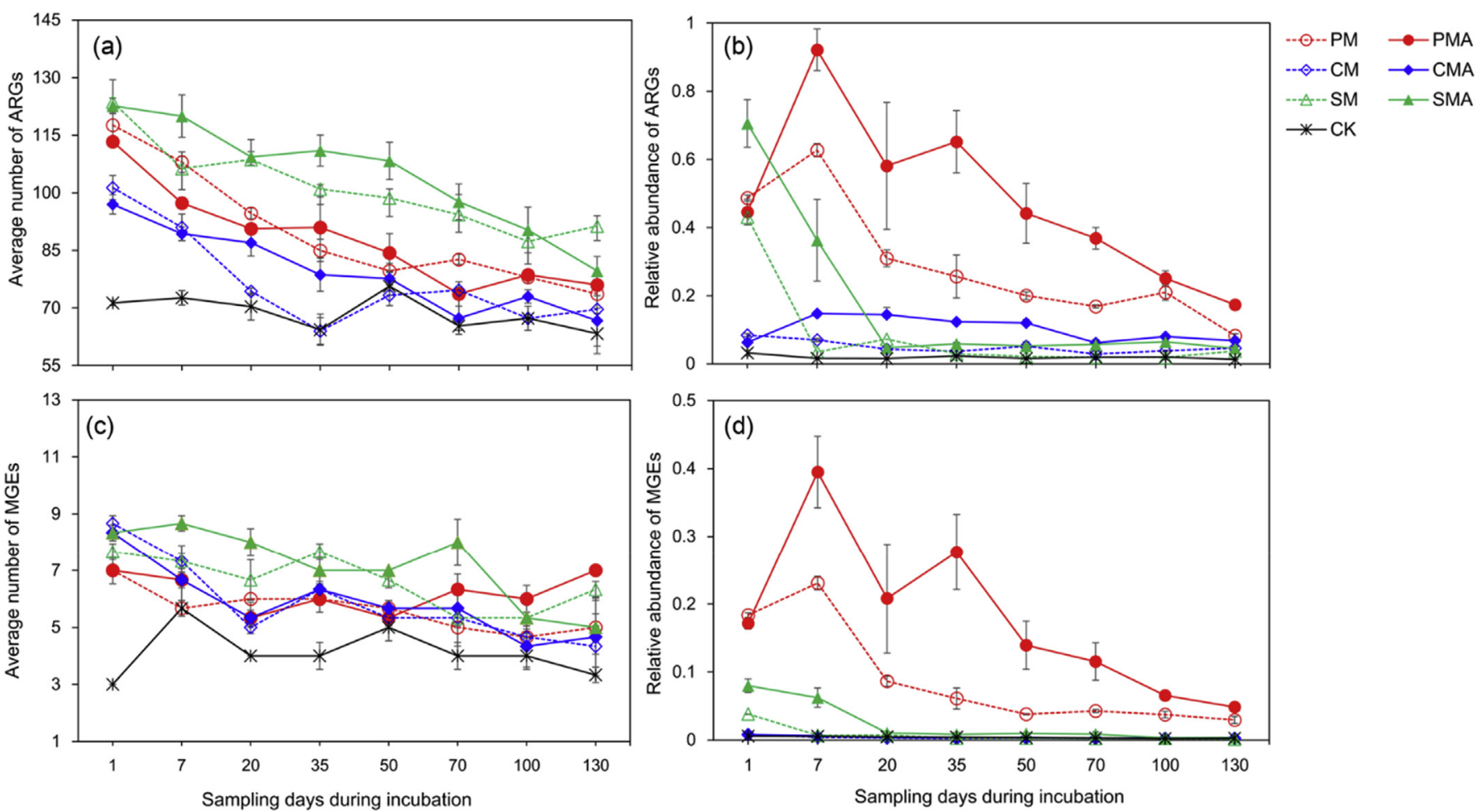

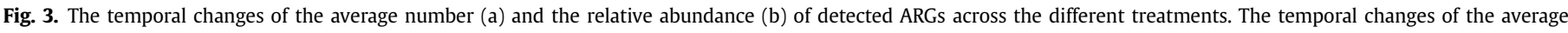

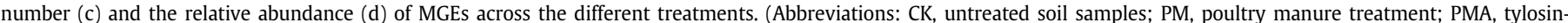

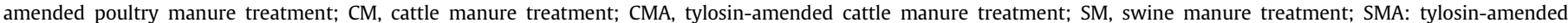
swine manure treatment; UPM, untreated poultry manure; UCM, untreated cattle manure; USM, untreated swine manure).

the incubation (Fig. 3c), where the relative abundances of MGEs exhibited highly similar temporal patterns to ARGs across different treatments (Fig. 3d). No significant impacts of cattle manure application on the relative and absolute abundances of soil MGEs were observed for both treatments with or without tylosin addition (Fig. 3d and Fig. S3). Pearson's correlation analysis revealed that the relative abundance of total ARGs was significantly correlated with that of total transposase genes $(P<0.001)$ and total integrase genes $(P<0.001)$ (Fig. 4). The abundances of the eight major classes of ARGs were also significantly and positively correlated with total transposase genes and total integrase genes, except MLSB and vancomycin resistance genes (Table 1 ).

\subsection{Temporal changes of individual subtypes of ARGs}

The distribution and temporal changes of individual ARG subtypes across different treatments were assessed at a higher resolution level in a heatmap based on the log-transformed relative abundance of each ARG subtypes (Fig. 5). The untreated soil samples showed the lowest detection frequencies of ARGs, which

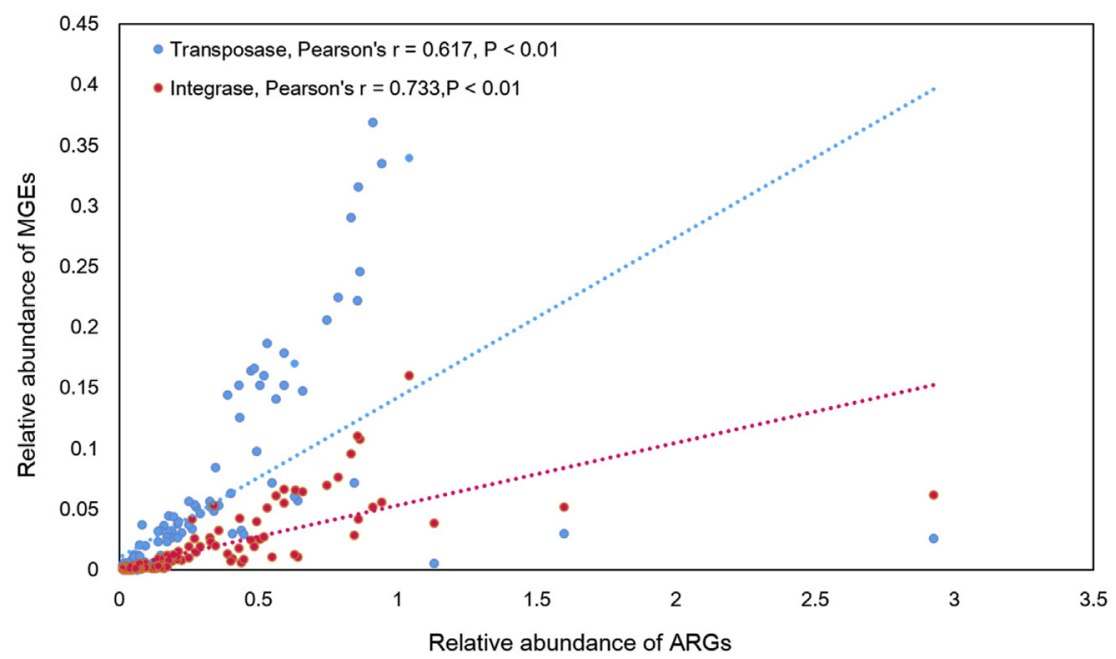

Fig. 4. Correlation between the relative abundance of total ARGs and the relative abundances of transposase genes or integrase genes. 
Table 1

Pearson correlations between the relative abundance of ARGs and MGEs in all the samples.

\begin{tabular}{lll}
\hline & \multicolumn{2}{l}{ Relative abundance of MGEs } \\
\cline { 2 - 3 } & Transposase & Integrase \\
\hline Total ARGs & $\mathbf{0 . 6 1 7}(<\mathbf{0 . 0 0 1})$ & $\mathbf{0 . 7 3 3}(<\mathbf{0 . 0 0 1})$ \\
Aminoglycoside & $\mathbf{0 . 9 2 6}(<\mathbf{0 . 0 0 1})$ & $\mathbf{0 . 8 2 6}(<\mathbf{0 . 0 0 1})$ \\
B-lactamase & $\mathbf{0 . 2 3}(<\mathbf{0 . 0 0 1})$ & $\mathbf{0 . 4 7 7}(<\mathbf{0 . 0 0 1})$ \\
FCA & $\mathbf{0 . 4 8 2}(<\mathbf{0 . 0 0 1})$ & $\mathbf{0 . 4 7 3}(<\mathbf{0 . 0 0 1})$ \\
MLSB & $0.242(0.088)$ & $\mathbf{0 . 2 3 9}(<\mathbf{0 . 0 0 1})$ \\
Multidrug & $\mathbf{0 . 8 8 5}(<\mathbf{0 . 0 0 1})$ & $\mathbf{0 . 9 4 3}(<\mathbf{0 . 0 0 1})$ \\
Sulfonamide & $\mathbf{0 . 8 4 0}(\mathbf{0 . 0 1 5})$ & $\mathbf{0 . 1 9 4}(\mathbf{0 . 0 0 1})$ \\
Tetracycline & $\mathbf{0 . 3 0 5}(<\mathbf{0 . 0 0 1})$ & $\mathbf{0 . 4 8 6}(<\mathbf{0 . 0 0 1})$ \\
Vancomycin & $\mathbf{0 . 2 6 9}(<\mathbf{0 . 0 0 1})$ & $0.338(0.072)$ \\
Other & $\mathbf{0 . 5 1 4}(<\mathbf{0 . 0 0 1})$ & $\mathbf{0 . 1 9 4}(\mathbf{0 . 0 0 1})$ \\
\hline
\end{tabular}

Values showed in the table are the r-values derived from the Pearson analysis, and the $P$-values are showed in the brackets. The bold numbers represent significant correlations $(P<0.05)$.

remained largely stable over time during the incubation. In general, manure application resulted in obvious increases in the relative abundances of a majority of ARG subtypes. Compared with the soils treated with manure only, the manure spiked with tylosin treatments further increased the relative abundances of all the major classes of ARGs (Fig. 5). The untreated poultry and swine manure samples had more abundant ARGs than untreated cattle manure samples, and therefore amendment of poultry and swine manure can result in a higher level of ARGs in soils during the microcosm incubation (Fig. 5). The non-metric multidimensional scaling (NMDS) ordination based on the Bray-Curtis dissimilarity matrices revealed that soil samples treated with different animal manures clustered separately, and samples treated with both manure and tylosin tended to separate from those treated with manure only (Fig. 6).

These ARGs showed different temporal patterns during the incubation (Fig. 5): (i) Some genes conferring resistance to aminoglycoside (aacC4, aadA-01, aadA-02, aadA-1-02, aadA2-01, aadA202), FCA, MLSB (ermY, lnuA-01), multidrug (oprD) and tetracycline (tetD-02), which were present in untreated soil samples, became more abundant in soils treated with manure and manure spiked with tylosin, especially for the poultry and swine manure treatments; (ii) Some genes conferring resistance to multidrug (yceL/ $m d t H-03$, yidY/mdtL-01, yidY/mdtL-02) and tetracycline (tetA-02, tetB-01, tetB-02, tetC-01), which were absent in untreated soil samples, appeared in manure and tylosin treated samples, and persisted until the end of the incubation; (iii) Several multidrug resistance genes (qacH-01, qacH-02, rarD-02, tolC-01, tolC-02 and tolC-03) and vancomycin resistance genes (vanRA-02, vanRB) were abundant in three types of manures, however, decreased over time in all the manured soils and became undetected at the end of the incubation; (iv) Most $\beta$-lactam resistant genes kept relatively unchanged in soils amended with manure in the presence or absence of tylosin.

\subsection{Co-occurrence patterns among ARGs and MGEs}

The network analysis was performed to explore the cooccurrence patterns of ARGs and MGEs based on strong $(\rho>0.8)$ and significant $(P<0.05)$ correlations. The resultant network was composed of 73 nodes (ARG subtypes) and 209 edges (pairwise correlations) (Fig. 7a), and could be clearly separated into six modules (Fig. 7b). Each module consisted of different types of ARGs, except module IV which included exclusively five tetracycline resistance genes. The most densely connected node in each module was defined as the 'hub', for example, the 'tolC-03' (multidrug resistance) and 'aadA2-01' (aminoglycoside resistance) were the hubs for modules I and II, respectively (Fig. 7b). The module III had three equivalent hub genes $v g b-01, a c r A-05$ and $y i d Y / m d t L-01$. It has been suggested that hubs could be regarded as indicator ARGs to represent the quantity of other co-occurring ARGs in the same module (Li et al., 2015). Interestingly, the intI1 gene (belonging to MGEs) had intensive connections with multiple ARGs which can potentially confer resistance to multiple classes of antibiotics (Fig. 7a).

\section{Discussion}

\subsection{Poultry and swine manures might have stronger selection pressure on soil resistome than cattle manure}

Animal manure has been long regarded as an important reservoir of ARGs, no matter whether or not the animals have been treated with antibiotics (Heuer et al., 2011; Udikoviv-Kolic et al., 2014; Hu et al., 2016a). Land application of animal manure, as a common agricultural practice, may introduce the inflow of a large amount of fecal microbiome including antibiotic resistant bacteria and potential human pathogens into the soil and groundwater environments (Chee-Sanford et al., 2009; Heuer et al., 2011; Wang et al., 2015). Functional metagenomic analysis revealed that manure-derived ARGs can account for up to $70 \%$ of the total ARGs in soils following manure application (Su et al., 2014). Swine, cattle, and poultry manures are three major types of widely-used organic fertilizers worldwide, but no studies have systematically compared their effects on the temporal patterns of soil ARGs. In this study, we found that the tested swine manure harboured the highest diversity and abundance of ARGs, followed by poultry manure, while the diversity and abundance of ARGs in cattle manure were significantly lower than the other two manure types $(P<0.05)$. This result supported previous findings that animal manure is a "hotspot" for environmental contamination with ARGs (Chee-Sanford et al., 2009; Heuer and Smalla, 2007) even from antibiotic-free animals (Jackson et al., 2004; Looft et al., 2012; Udikoviv-Kolic et al., 2014; Hu et al., 2016a). Our findings imply that attentions might be particularly paid to swine and poultry manures which need to be properly treated, i.e. by composting (Gou et al., 2017), to reduce the levels of ARGs before land application.

Although the untreated agricultural soil examined in this study had a diverse array of ARGs (Fig. 1b), its ARG abundances were significantly lower than all three types of animal manures. Animal manure has been considered as a significant reservoir of ARGs, even if the animals have never been treated with antibiotics (UdikovivKolic et al., 2014; Hu et al., 2016a), suggesting that antibiotic resistance is intrinsic in animal guts. Therefore, manure application can dramatically increase the abundance of soil ARGs (Fig. 2b and d), and can also introduce a number of unique manure-derived ARGs into soil. For example, the tested swine manure is highly abundant in tetracycline, sulfonamide, MLSB, and $\beta$-lactam resistance genes, while the tested poultry manure is abundant in multidrug, and aminoglycoside resistance genes, and thus application of these two manures substantially enhanced abundances of these genes, resulting in contrasting profiles of ARGs across the different manure treatments (Fig. 2). Cattle manure application had relatively slight impacts on soil ARG diversity compared to poultry and swine manures (Fig. 1), but still significantly increased soil ARG abundances, in particular, the abundances of aminoglycoside and multidrug resistance genes (Fig. 2). Therefore, different sources of animal manures could manipulate soil resistome in varying magnitudes, and different soil ARG profiles in manured soils would differ in their consequences for soil and public health. The differences in ARG profiles in the tested three manure sources could be 


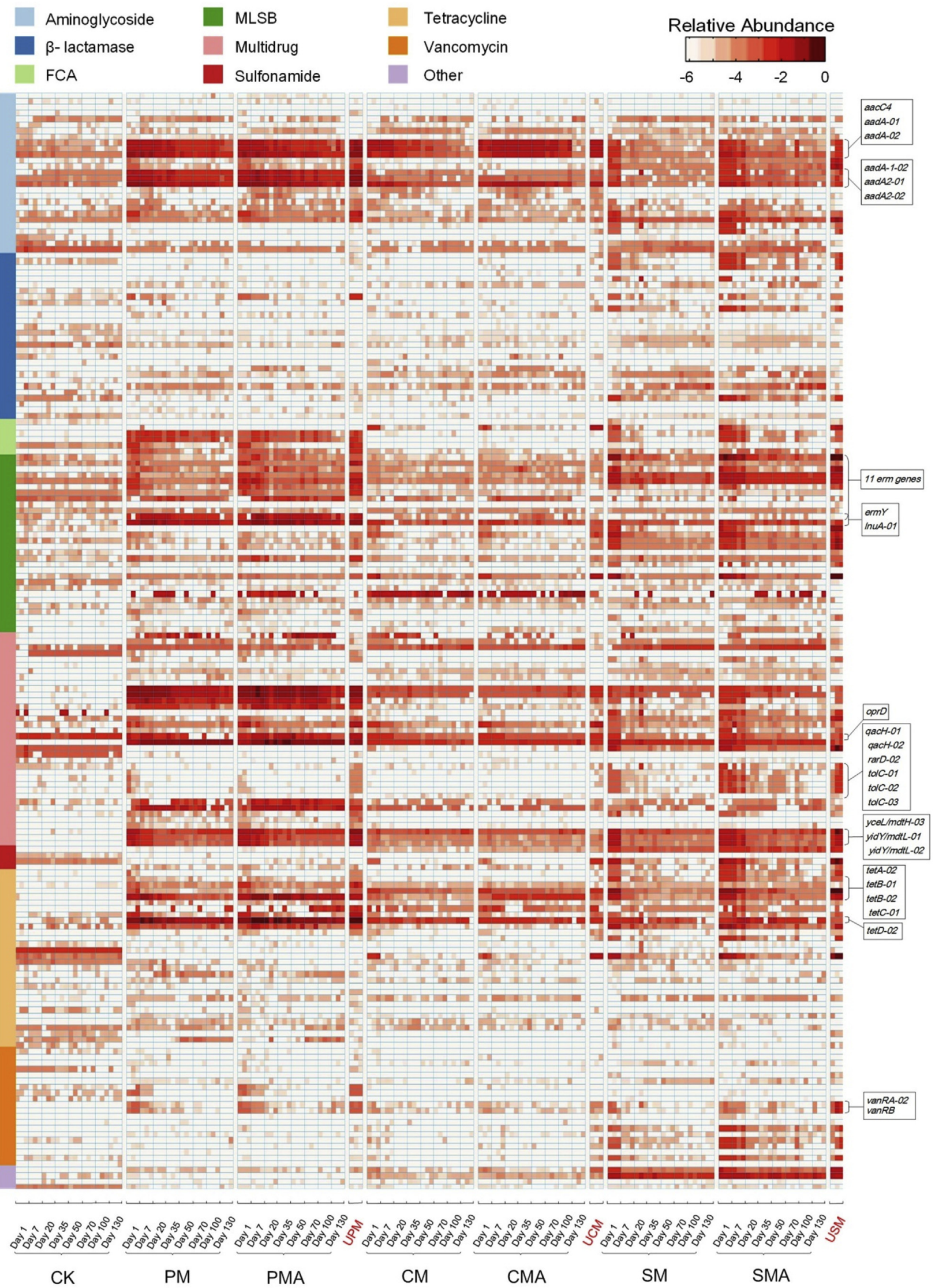

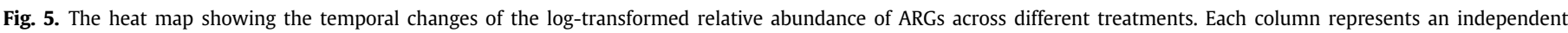

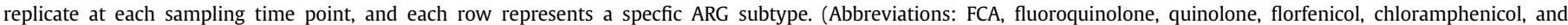

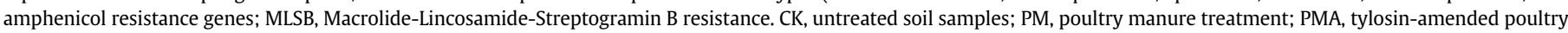

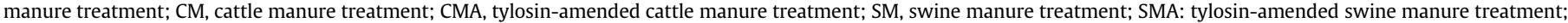
UPM, untreated poultry manure; UCM, untreated cattle manure; USM, untreated swine manure). 


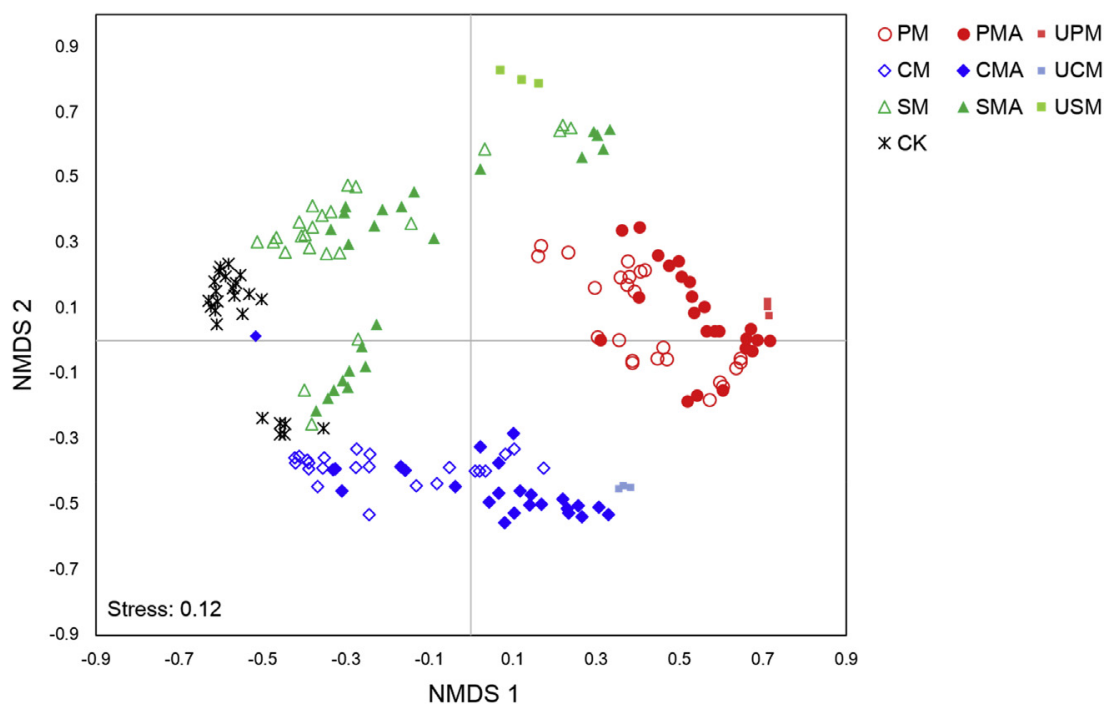

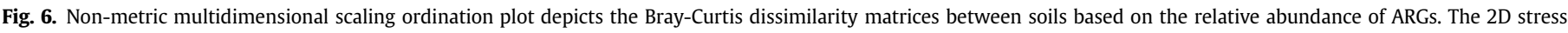

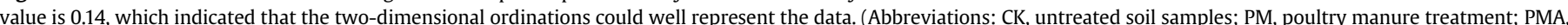

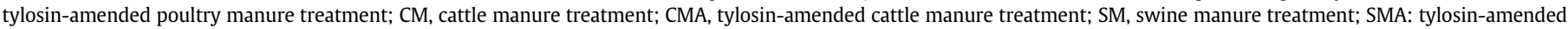
swine manure treatment; UPM, untreated poultry manure; UCM, untreated cattle manure; USM, untreated swine manure).

attributed to the dietary, antibiotic use history, and indigenous gut resistome of the animals.

Despite the different ARG profiles in soils treated with different animal manure sources, the diversity and abundance of ARGs in all manured soils gradually decreased over time, except the relative abundance of ARGs in cattle manure-treated samples (Fig. 3). Notably, the relative abundance of ARGs in soils treated with swine manure with or without tylosin dramatically declined to approach the background levels of ARGs in untreated soils within 20 or 7 days, respectively, whereas ARGs in poultry manure-treated soils persisted in the entire course of incubation and were still more abundant than background levels at day 130 (Fig. 3b). These findings have implications for agricultural management practice from the perspective of minimizing antibiotic resistance: raw swine manure might be applied to the field one month before the vegetable harvest, but raw poultry and cattle manures might be used at least three $\sim$ four months before the final harvest, with poultry manure having the highest risk of ARGs spreading based on the results generated from this microcosm study.

The time-course reduction of ARGs following manure application may be explained by the gradual out-competition of manurederived bacteria by the soil indigenous microbiomes, and the different conditions between animal gut and soil environments (Chee-Sanford et al., 2009; Hu et al., 2016a). A number of ARGs, such as aacC4 and aadA genes (aminoglycoside resistance); ermY and InuA-01 genes (MLSB resistance); oprD gene (multidrug resistance); and tetD-02 gene (tetracycline resistance), persisted until the end of the incubation, particularly in the poultry manure treatments (Fig. 5). Some genes including the tolC-03 (multidrug resistance) and aadA2-01 (aminoglycoside resistance), identified as the "hubs" by the network analysis (Fig. 7), were densely connected other ARGs and MGEs during the microcosm incubation. There has been evidence that such "hub" genes can be used as indicator genes to monitor the temporal changes of other co-occurring ARGs in the same module of networks (Li et al., 2015). Therefore, these ARGs might have the highest potential to be captured by human pathogens and pose a threat to human health.

Despite of these findings, it should be noted that (1) the microcosm incubation approach used in this study has its own limitations, and an actual field is highly complex and it is difficult to reproduce all its features (e.g., solar radiation, daytime and night time temperatures, oxygen availability, and other environmental factors influencing the persistence of ARGs); (2) the soil used in this study is a very sandy soil, which might limit the extrapolation of the findings to other soils with contrasting soil texture and environmental conditions; (3) the level of tylosin spiked to the microcosms was several orders of magnitude higher the levels of tylosin commonly measured in natural environments (Wegst-Uhrich et al., 2014), which might exert stronger selection pressure on soil ARGs; (4) the tylosin used in this study was spiked to animal manures rather than administered to animals in feed and excreted in manure. Tylosin can interact with feces in the intestinal tract of animals before being excreted and then continues to interact with manure until the land application of animal manure. There has been evidence that the dissipation rate of antibiotics directly spiked to manure was different from that of antibiotics consumed and excreted by cattle (Amarakoon et al., 2016). Therefore, the effect of directly-spiked antibiotics and animal-excreted antibiotic residues on soil resistome can be highly different.

\subsection{Antibiotic tylosin amendment imposed an additional selection pressure on ARGs}

The use of human-made antibiotics in livestock production and human medicines was considered as the major reason for the exponentially increasing ARGs in environmental samples since 1940 (Knapp et al., 2010). Many previous studies have investigated the effects of manure collected from antibiotic-treated animals on the composition and mobility potential of ARGs in natural settings (Jechalke et al., 2013, 2014a; Luby et al., 2016). No studies have, however, provided comprehensive insights into the impacts of the antibiotic tylosin on the temporal patterns of a wide spectrum of soil ARGs. Tylosin belongs to macrolide subclass in the MLSB class, which was the most frequently detected antibiotic resistance type in this experiment (Fig. 1a). Although it is supposed that tylosin has a short half-life of a couple of days in soils (Kolz et al., 2005), its impacts would last for a long period after its decomposition, as demonstrated by the persistence of ARGs for many years in the 


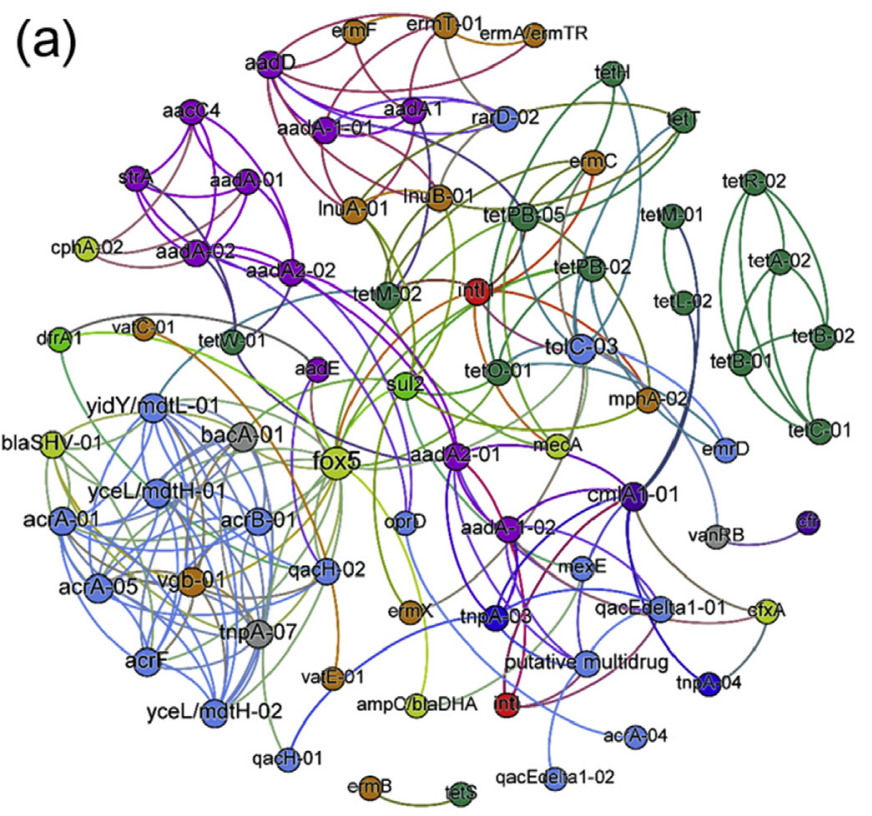

Multidrug $\bigcirc$ Tetracycline $\bigcirc$ mLSB $\bigcirc$ Aminoglycoside $\bigcirc$-lactamase
IS6 Group $\bigcirc$ FCA $\bigcirc$ sulfonamide $\bigcirc$ Others $\bigcirc$ MGEs/Integrase

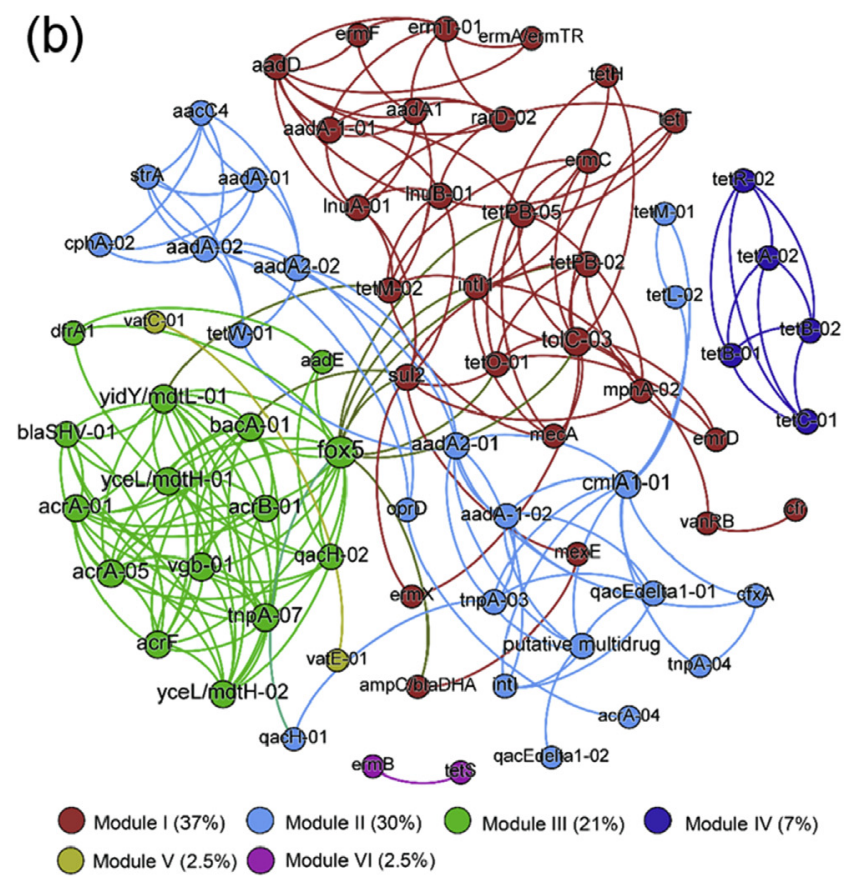

Fig. 7. The networks depicting the co-occurrence patterns among the detected ARGs and MGEs. The nodes coded with different colors represent different classes of ARGs (a) and different modules (b). The edges connecting nodes correspond to statistically significant correlations between nodes. Node size is proportional to the number of connections between nodes (degree). (For interpretation of the references to colour in this figure legend, the reader is referred to the web version of this article.)

absence of the corresponding antibiotic (Johnsen et al., 2009). Therefore, it is not surprising to observe increased abundances of MLSB resistance genes in tylosin-spiked poultry and swine manure-treated soils until the end of the incubation (Fig. 2), indicating a strong selection effect of tylosin on the MLSB resistance type.

Erythromycin ribosome methylation (erm) genes encode tylosin resistance by reducing the ability of tylosin from binding to the $50 \mathrm{~S}$ ribosomal subunit (Weisblum, 1998), and have been detected in microbial taxa including staphylococci, Enterococcus, Stretococccus, and Bacillus cereus (Jensen et al., 2002). For example, three main erm genes have been described in staphylococci: erm(A) gene is located on transposon Tn554, erm(B) gene on transposon Tn551 and erm(C) gene on a plasmid (Saribas et al., 2006), suggesting the HGT potential of the erm genes. We detected $11 \mathrm{erm}$ genes in this study, and these genes were obviously more abundant in the tylosinamended treatments, especially in the poultry manure and swine manure treatments (Fig. 5). In the swine manure treatments, the relative abundances of erm genes were significantly higher in the tylosin-amended treatments compared with the tylosin-absent treatments (Fig. S4, $P<0.05$ ). Therefore, the presence of tylosin in the tested swine manures can impose strong selection pressure on soil microbiome, and might increase the propagation of tylosinresistant bacteria.

Beyond the selection effect of tylosin on MLSB resistance genes, it is interesting to find that ARGs encoding resistance to almost all the major classes of ARGs increased for tylosin-amended treatments when compared with manured soils without tylosin (Fig. 2). This non-targeted selection phenomenon might be explained by the co-selection mechanisms (Hu et al., 2016b) in which genes encoding resistance to different antibiotics may reside in the same MGEs (plasmid, integrin, or transposon) or single genes can encode resistance to various classes of ARGs. As shown in the cooccurrence network (Fig. 7), genes conferring resistance to different categories of antibiotics were shared in the same module, suggesting that these ARGs might be carried by the same bacterial cells or MGEs, and they might change and transfer together under the selection pressure imposed by tylosin. Therefore, our findings caution that the addition of tylosin can select for a broad range of ARGs apart from MLSB resistance, and elongate the lifespan of ARGs throughout the whole incubation period (in poultry and swine manured soils).

\subsection{The HGT potential of ARGs in soils treated with manure and tylosin}

The HGT of ARGs among environmental bacteria of different taxa is an important pathway for resistance dissemination and the subsequent acquisition of resistance by human pathogens and commensals (Heuer et al., 2011; Forsberg et al., 2012). Previous studies have demonstrated that manure addition may promote the HGT potential of soil ARGs, because manure generally contains high loads of broad-host-range plasmids, which are important vectors of ARGs (Heuer et al., 2011). Under the selection pressure of antibiotic, fecal microbes that cannot persist in the soil environment may transfer ARGs to resident soil bacteria via HGT mediated by MGEs (Heuer et al., 2011). Once being transferred, the resistance traits could persist in natural settings because native bacteria are generally better adapted to the soil environment (Chee-Sanford et al., 2009). Therefore, amendment of both manure and antibiotic could interactively promote the HGT potential of ARGs in soils.

In this study, the abundances of both integrase and transposase genes were significantly and positively correlated with those of total ARGs and individual ARG types across all the treatments (Table 1), indicating that there is a potential for HGT of ARGs in the tested agricultural soils after manure/antibiotic application. Particularly, the relative abundances of MGEs were significantly higher in poultry manure treatment compared with untreated soil 
samples and other manure treatments, and this enrichment was even more remarkable in the presence of tylosin (Fig. 2). Therefore, ARGs in the poultry manure-treated soils may have greater HGT potential than those in other treatments. In addition, we found that the intI1 gene (belonging to MGEs) had intensive connections with multiple ARGs conferring resistance to multiple classes of antibiotics in the co-occurrence network, suggesting that class 1 integron may play important roles in dissemination of these co-occurring ARGs, which is also reported in previous studies (Gillings et al., 2015). Altogether, the enormous diversity of ARGs and MGEs in manure-treated agricultural soils might provide a high likelihood of dispersal and HGT of soil ARGs, however the actual frequencies of HGT should be tested in future studies.

\section{Conclusions}

In conclusion, by using HT-qPCR ARG arrays in this study, we provide evidence that the tested poultry and swine manures have stronger impacts on the diversity, abundance and HGT potential of a wide spectrum of soil ARGs than the tested cattle manure in soil microcosm experiments. Such effects of manure addition on soil resistome were enhanced by addition of the antibiotic tylosin, which selected for increased bacterial resistance to multiple categories of antibiotics and prolonged the persistence of ARGs during the soil microcosm incubation. Our findings have important implications for public health if these enriched ARGs following manure application can be transferred into the food chain through human consumption of the harvested vegetables, and necessitate the appropriate treatment of raw animal manures (especially poultry and swine manures) to minimise the dissemination of environmental ARGs. Future studies by including multiple animal manures of each type are required to verify if the temporal patterns of ARGs observed in this study are broadly applied to a wide variety of soil types.

\section{Acknowledgements}

This work was financially supported by Australian Research Council (DP170103628, DE150100870) and the Australia-China Joint Research Centre (ACSRF48165).

\section{Appendix A. Supplementary data}

Supplementary data related to this article can be found at https://doi.org/10.1016/j.envpol.2017.09.074.

\section{References}

Amarakoon, I.D., Zvomuya, F., Sura, S., Larney, F.J., Cessna, A.J., Xu, S., McAllister, T.A. 2016. Dissipation of antimicrobials in feedlot manure compost after oral administration versus fortification after excretion. J. Environ. Qual. 45, 503-510.

Apley, M.D., Bush, E.J., Morrison, R.B., Singer, R.S., Snelson, H., 2012. Use estimates of in-feed antimicrobials in swine production in the United States. Foodborne Pathog. Dis. 9, 272-279.

Bastian, M., Heymann, S., Jacomy, M., 2009. Gephi: an open source software for exploring and manipulating networks. ICWSM 8, 361-362.

Bibbal, D., Dupouy, V., Ferré, J.P., Toutain, P.L., Fayet, O., Prere, M.F., BousquetMélou, A., 2007. Impact of three ampicillin dosage regimens on selection of ampicillin resistance in Enterobacteriaceae and excretion of blaTEM genes in swine feces. Appl. Environ. Microbiol. 73, 4785-4790.

Byrne-Bailey, K., Gaze, W.H., Kay, P., Boxall, A.B., Hawkey, P.M., Wellington, E.M., 2009. Prevalence of sulfonamide resistance genes in bacterial isolates from manured agricultural soils and pig slurry in the United Kingdom. Antimicrob. Agents Chemother 53, 696-702.

Chee-Sanford, J.C., Mackie, R.I., Koike, S., Krapac, I.G., Lin, Y.F., Yannarell, A.C. Maxwell, S., Aminov, R.I., 2009. Fate and transport of antibiotic residues and antibiotic resistance genes following land application of manure waste J. Environ. Qual. 38, 1086-1108.

Dominey-Howes, D., Labbate, M., 2014. The antimicrobial resistance crisis: causes, consequences, and management. Front. Public Health 2, 145.
Forsberg, K.J., Reyes, A., Wang, B., Selleck, E.M., Sommer, M.O.A., Dantas, G., 2012. The shared antibiotic resistome of soil bacteria and human pathogens. Science 337, 1107-1111.

Garder, J.L., Moorman, T.B., Soupir, M.L., 2014. Transport and persistence of tylosinresistant enterococci, genes, and tylosin in soil and drainage water from fields receiving swine manure. J. Environ. Qual. 43, 1484-1493.

Gelband, H., Molly Miller, P., Pant, S., Gandra, S., Levinson, J., Barter, D., White, A., Laxminarayan, R., 2015. The state of the world's antibiotics 2015. Wound Heal. South. Afr. 8, 30-34.

Gillings, M.R., Gze, W.H., Pruden, A., Smalla, K., Tiedje, J.M., Zhu, Y.G., 2015. Using the class 1 integron-integrase gene as a proxy for anthropogenic pollution. ISME J. 9, 1269-1279.

Gou, M., Hu, H.W., Zhang, Y.J., Wang, J.T., Hayden, H., Tang, Y.Q., He, J.Z., 2017. Aerobic composting reduces antibiotic resistance genes in cattle manure and the resistome dissemination in agricultural soils. Sci. Tol. Environ. 612, $1300-1310$.

Han, X.M., Hu, H.W., Shi, X.Z., Wang, J.T., Han, L.L., Chen, D., He, J.Z., 2016. Impacts of reclaimed water irrigation on soil antibiotic resistor in urban parks of Victoria, Australia. Environ. Pollut. 211, 48-57.

Heuer, H., Schmitt, H., Smalla, K., 2011. Antibiotic resistance gene spread due to manure application on agricultural fields. Curr. Opin. Microbiol. 14, 236-243.

Heuer, H., Smalla, K., 2007. Manure and sulfadiazine synergistically increased bacterial antibiotic resistance in soil over at least two months. Environ. Microbiol. 9, 657-666.

Hölzel, C.S., Harms, K.S., Küchenhoff, H., Kunz, A., Müller, C., Meyer, K., Schwaiger, K., Bauer, J., 2010. Phenotypic and genotypic bacterial antimicrobial resistance in liquid pig manure is variously associated with contents of tetracyclines and sulfonamides. J. Appl. Microbiol. 108, 1642-1656.

Hu, H.W., Han, X.M., Shi, X.Z., Wang, J.T., Han, L.L., Chen, D., He, J.Z., 2016a. Temporal changes of antibiotic resistance genes and bacterial communities in two contrasting soils treated with cattle manure. FEMS Microbiol. Ecol. 92 fiv196.

Hu, H.W., Wang, J.T., Li, J., Li, J.J., Ma, Y.B., Chen, D., He, J.Z., 2016b. Field-based evidence for copper contamination induced changes of antibiotic resistance in agricultural soils. Environ. Microbiol. 18, 3896-3909.

Hu, H.W., Wang, J.T., Li, J., Shi, X.Z., Ma, Y., Chen, D., He, J.Z., 2017. Long-term nickel contamination increases the occurrence of antibiotic resistance genes in agricultural soils. Environ. Sci. Technol. 51, 790-800.

Jackson, C.R., Fedorka-Cray, P.J., Barrett, J.B., Ladely, S.R., 2004. Effects of tylosin use on erythromycin resistance in enterococci isolated from swine. Appl. Environ. Microbiol. 70, 4205-4210.

Jechalke, S., Focks, A., Rosendahl, I., Groeneweg, J., Siemens, J., Heuer, H., Smalla, K. 2014a. Structural and functional response of the soil bacterial community to application of manure from difloxacin-treated pigs. FEMS Microbiol. Ecol. 87, $78-88$.

Jechalke, S., Heuer, H., Siemens, J., Amelung, W., Smalla, K., 2014b. Fate and effects of veterinary antibiotics in soil. Trends Microbiol. 22, 536-545.

Jechalke, S., Kopmann, C., Rosendahl, I., Groeneweg, J., Weichelt, V., Krögerrecklenfort, E., Brandes, N., Nordwig, M., Ding, G.C., Siemens, J., 2013. Increased abundance and transferability of resistance genes after field application of manure from sulfadiazine-treated pigs. Appl. Environ. Microbiol. 79, $1704-1711$.

Jensen, L.B., Agersø, Y., Sengeløv, G., 2002. Presence of erm genes among macrolideresistant Gram-positive bacteria isolated from Danish farm soil. Environ. Int. 28, $487-491$.

Johnsen, P.J., Townsend, J.P., Bøhn, T., Simonsen, G.S., Sundsfjord, A., Nielsen, K.M., 2009. Factors affecting the reversal of antimicrobial-drug resistance. Lancet Infect. Dis 9, 357-364.

Johnson, T.A., Stedtfeld, R.D., Wang, Q., Cole, J.R., Hashsham, S.A., Looft, T., et al., 2016. Clusters of antibiotic resistance genes enriched together stay together in swine agriculture. mBio 7, e02214-e02215.

Junker, B., Schreiber, F., 2008. Correlation Networks. Analysis of Biological Networks.

Kolz, A., Moorman, T., Ong, S.K., Scoggin, K., Douglass, E., 2005. Degradation and metabolite production of tylosin in anaerobic and aerobic swine-manure lagoons. Water Environ. Res. 77, 49-56.

Knapp, C.W., Dolfing, J., Ehlert, P.A., Graham, D.W., 2010. Evidence of increasing antibiotic resistance gene abundances in archived soils since 1940. Environ. Sci. Technol. 44, 580-587.

Koch, B.J., Hungate, B.A., Price, L.B., 2017. Food-animal production and the spread of tha antibiotic resistance: the role of ecology. Front. Ecol. Environ. 15, 309-318.

Li, B., Yang, Y., Ju, F., Guo, F., Tiedje, J.M., Zhang, T., 2015. Metagenomic and network analysis reveal wide distribution and co-occurrence of environmental antibiotic resistance genes. ISME J. 9, 2490-2502.

Looft, T., Johnson, T.A., Allen, H.K., Bayles, D.O., Alt, D.P., Stedtfeld, R.D., Sul, W.J., Stedtfeld, T.M., Chai, B., Cole, J.R., 2012. In-feed antibiotic effects on the swine intestinal microbiome. Proc. Natl. Acad. Sci. U.S.A. 109, 1691-1696.

Luby, E.M., Moorman, T.B., Soupir, M.L., 2016. Fate and transport of tylosin-resistant bacteria and macrolide resistance genes in artificially drained agricultural fields receiving swine manure. Sci. Tot. Environ. 550, 1126-1133.

Manaia, C.M., 2017. Assessing the risk of antibiotic resistance transimission from the environment to humans: non-direct proportionality between abundance and risk. Trends Microbiol. 25, 173-181.

Marti, R., Scott, A., Tien, Y.C., Murray, R., Sabourin, L., Zhang, Y., Topp, E., 2013. Impact of manure fertilization on the abundance of antibiotic-resistant bacteria and frequency of detection of antibiotic resistance genes in soil and on 
vegetable at harvest. Appl. Environ. Microbiol. 79, 5701-5709.

Muurinen, J., Stedtfeld, R., Karkman, A., Katariina, P., Tiejie, J., Virta, M., 2017. Influence of manure application on the environmental resistome under Finnish agricultural practice with restricted antibiotic use. Environ. Sci. Technol. 51, 5989-5999.

Peng, S., Wang, Y., Zhou, B., Lin, X., 2015. Long-term application of fresh and composted manure increase tetracycline resistance in the arable soil of eastern China. Sci. Tot. Environ. 506, 279-286.

Pruden, A., Pei, R., Storteboom, H., Carlson, K.H., 2006. Antibiotic resistance genes as emerging contaminants: studies in Northern Colorado. Environ. Sci. Technol. 40, 7445-7450.

Rossolini, G.M., Arena, F., Pecile, P., Pollini, S., 2014. Update on the antibiotic resistance crisis. Curr. Opin. Pharmacol. 18, 56-60.

Saribas, Z., Tunckanat, F., Pinar, A., 2006. Prevalence of erm genes encoding macrolide-lincosamide-streptogramin (MLS) resistance among clinical isolates of Staphylococcus aureus in a Turkish university hospital. Clin. Microbiol. Infect. 12, 797-799.

Sarmah, A.K., Meyer, M.T., Boxall, A.B., 2006. A global perspective on the use, sales, exposure pathways, occurrence, fate and effects of veterinary antibiotics (VAs) in the environment. Chemosphere 65, 725-759.

Schmittgen, T.D., Livak, K.J., 2008. Analyzing real-time PCR data by the comparative CT method. Nat. Protoc. 3, 1101-1108.

Smalla, K., Heuer, H., Götz, A., Niemeyer, D., Krögerrecklenfort, E., Tietze, E., 2000 Exogenous isolation of antibiotic resistance plasmids from piggery manure slurries reveals a high prevalence and diversity of IncQ-like plasmids. Appl. Environ. Microbiol. 66, 4854-4862.

Soffer, N., Zaneveld, J., Vega Thurber, R., 2015. Phage-bacteria network analysis and its implication for the understanding of coral disease. Environ. Microbiol. 17, 1203-1218.

Su, J.Q., Wei, B., Xu, C.Y., Qiao, M., Zhu, Y.G., 2014. Functional metagenomic characterization of antibiotic resistance genes in agricultural soils from China.
Environ. Int. 65, 9-15.

Suzuki, M.T., Taylor, L.T. Delong, E.F. 2000. Quantitative analysis of small-subunit rRNA genes in mixed microbial populations via 5'-nuclease assays. Appl. Environ. Microbiol. 66, 4605-4614.

Tenover, F.C., 2006. Mechanisms of antimicrobial resistance in bacteria. Am. J. Med. 119, S3-S10.

Thanner, S., Drissner, D., Walsh, F., 2013. Antibiotic resistance in agriculture. mBio 7 e02227-15.

Thomas, C.M., Nielsen, K.M., 2005. Mechanisms of, and barriers to, horizontal gene transfer between bacteria. Nat. Rev. Microbiol. 3, 711-721.

Udikoviv-Kolic, N., Wichmann, F., Broderick, N.A., Handelsman, J., 2014. Bloom of resident antibiotic-resistant bacteria in soil following manure fertilization. Proc. Natl. Acad. Sci. U.S.A. 111, 15202-15207.

Wang, F.H., Qiao, M., Chen, Z., Su, J.Q., Zhu, Y.G., 2015. Antibiotic resistance genes in manure-amended soil and vegetables at harvest. J. Hazard. Mater 299, 215-221.

Weisblum, B., 1998. Macrolide resistance. Drug resist. Update 1, 29-41.

Wegst-Uhrich, S., Navarro, D.A.G., Zimmerman, L., Aga, D.S., 2014. Assessing antibiotic sorption in soil: a literature review and new case studies on sulfonamides and macrolides. Chem. Cent. J. 8, 5.

World Health Organization, 2014. Antibiotic Resistance: Global Report on Surveillance. World Health Organization, Geneva, Switzerland.

Zhang, X.X., Zhang, T., 2011. Occurrence, abundance, and diversity of tetracycline resistance genes in 15 sewage treatent plants across China and other global locations. Environ. Sci. Technol. 45, 2598-2604.

Zhu, B., Chen, Q., Chen, S., Zhu, Y., 2017. Does organically produced lettuce harbor higher abundance of antibiotic resistance genes than conventionally produced? Environ. Int. 98, 152-159.

Zhu, Y.G., Johnson, T.A., Su, J.Q., Qiao, M., Guo, G.X., Stedtfeld, R.D., et al., 2013. Diverse and abundant antibiotic resistance genes in Chinese swine farms. Proc. Natl. Acad. Sci. U. S. A. 110, 3435-3440. 